\title{
An aggrecan fragment drives osteoarthritis pain through Toll-like receptor 2
}

Rachel E. Miller, ${ }^{1}$ Shingo Ishihara, ${ }^{1}$ Phuong B. Tran, ${ }^{1}$ Suzanne B. Golub, ${ }^{2}$ Karena Last, ${ }^{2}$ Richard J. Miller, ${ }^{3}$ Amanda J. Fosang, ${ }^{2}$ and Anne-Marie Malfait ${ }^{1}$

'Department of Internal Medicine, Division of Rheumatology, Rush University Medical Center, Chicago, Illinois, USA. 2University of Melbourne Department of Paediatrics, Murdoch Children's Research Institute, Royal Children's Hospital, Parkville, Victoria, Australia. ${ }^{3}$ Department of Pharmacology, Northwestern University, Chicago, Illinois, USA

\begin{abstract}
Pain is the predominant symptom of osteoarthritis, but the connection between joint damage and the genesis of pain is not well understood. Loss of articular cartilage is a hallmark of osteoarthritis, and it occurs through enzymatic degradation of aggrecan by cleavage mediated by a disintegrin and metalloproteinase with thrombospondin motif 4 (ADAMTS-4) or ADAMTS- 5 in the interglobular domain $\left(E^{373}-374 A\right)$. Further cleavage by MMPs $\left(N^{341}{ }^{342} \mathrm{~F}\right)$ releases a 32 -aminoacid aggrecan fragment (32-mer). We investigated the role of this 32-mer in driving joint pain. We found that the 32-mer excites dorsal root ganglion nociceptive neurons, both in culture and in intact explants. Treatment of cultured sensory neurons with the 32-mer induced expression of the proalgesic chemokine CCL2. These effects were mediated through TLR2, which we demonstrated was expressed by nociceptive neurons. In addition, intra-articular injection of the 32-mer fragment provoked knee hyperalgesia in WT but not TIr2-null mice. Blocking the production or action of the 32-mer in transgenic mice prevented the development of knee hyperalgesia in a murine model of osteoarthritis. These findings suggest that the aggrecan 32-mer fragment directly activates TLR2 on joint nociceptors and is an important mediator of the development of osteoarthritis-associated joint pain.
\end{abstract}

Conflict of interest: The authors have declared that no conflict of interest exists.

Submitted: June 14, 2017 Accepted: February 20, 2018 Published: March 22, 2018

\section{Reference information:} JCI Insight. 2018;3(6):e95704. https://doi.org/10.1172/jci. insight. 95704.

\section{Introduction}

An early event in the pathogenesis of osteoarthritis is enzymatic cleavage of the major cartilage proteoglycan aggrecan in the interglobular domain $\left(E^{373} \downarrow^{374} \mathrm{~A}\right)(1-3)$ by a disintegrin and metalloproteinase with thrombospondin motif $\underline{4}$ (ADAMTS-4) or ADAMTS-5 $(4,5)$. This results in loss of the bulk of the aggrecan molecule from the articular cartilage, and therefore, this cleavage step is critical for the development of osteoarthritis $(6,7)$. Accordingly, many pharmaceutical programs over the last 2 decades have focused on developing disease-modifying osteoarthritis drugs targeting ADAMTS-4 and ADAMTS-5 (8-10).

Once aggrecan is cleaved in the interglobular domain by ADAMTS-4/ADAMTS-5, the remaining $70-\mathrm{kDa} \mathrm{N}$-terminal aggrecan fragment is retained in the cartilage matrix and is subsequently cleaved by MMPs at $\mathrm{N}^{341} \downarrow^{342} \mathrm{~F}$, releasing a 32-amino acid fragment (32-mer, ${ }^{342} \mathrm{~F}-\mathrm{E}^{373}$ ) (11). This 32-mer has been identified in synovial fluid from osteoarthritis patients (12), and we have demonstrated that this fragment can promote proinflammatory signaling in chondrocytes, synovial fibroblasts, and peritoneal macrophages, through the activation of TLR2 (13)

TLR signaling has been implicated in a variety of neuroimmune processes $(14,15)$. Dorsal root ganglion (DRG) sensory neurons have been shown to express TLR3, TLR4, TLR5, TLR7, and TLR9 (1517). TLR2 has been shown to be expressed by satellite glial cells in the DRG, and by microglia, astrocytes, and neurons in the central nervous system $(15,18)$. In nerve injury models, Tlr2-null mice are partially protected from developing mechanical allodynia (19), macrophage infiltration into the DRG (20), and microglial activation in the spinal cord (19). Sensory neurons have been shown to respond to pathogen-associated molecular pattern molecules (PAMPs) such as lipopolysaccharide, dsRNA, and bacterial DNA motifs $(21,22)$, as well as to the damage-associated molecular pattern molecule (DAMP) HMGB-1 (23, 24). We recently reported that nociceptors can respond to DAMPs present in the osteoarthritic joint, such 
Table 1. Intracellular calcium responses in cultured DRG neurons

\begin{tabular}{lccc}
\hline & No. neuronal responses/total no. neurons & \% Responding & $\boldsymbol{P}^{\text {value }^{\mathrm{A}}}$ \\
WT: 32 -mer peptide $(3 \mu \mathrm{M})$ & $71 / 309$ & $23 \%$ & $P<0.0001$ \\
WT: Scrambled peptide $(3 \mu \mathrm{M})$ & $18 / 299$ & $6 \%$ & $5 \%$ \\
TIr2-null: 32 -mer peptide $(10 \mu \mathrm{M})$ & $3 / 57$ & & \\
${ }^{\mathrm{A}} \chi^{2}$ test was used to compare the number of responding and nonresponding neurons among these 3 groups. & \\
\hline
\end{tabular}

as S100A8 and $\alpha 2$-macroglobulin, by activating TLR4 expressed by joint nociceptors (16). Thus, it is likely that nociceptors might also sense and respond to other DAMPs, particularly those released from the cartilage extracellular matrix as a result of ongoing degradation.

Therefore, in this study we sought to investigate whether DRG neurons can express TLR2 and whether the 32-mer aggrecan fragment can directly excite DRG neurons through TLR2. Additionally, we tested whether this signaling pathway may play a role in osteoarthritis pain. We found that, indeed, DRG neurons express TLR2 and are able to respond to the 32-mer through this receptor. Furthermore, intra-articular injection of the 32-mer into the mouse knee induces acute knee hyperalgesia. Finally, in the destabilization of the medial meniscus (DMM) mouse model of osteoarthritis, inhibiting either the production or action of the 32-mer is sufficient to prevent knee hyperalgesia.

\section{Results}

We first examined the ability of the 32-mer to directly excite DRG neurons by examining its effects on mobilization of intracellular calcium $\left(\left[\mathrm{Ca}^{2+}\right]_{\mathrm{i}}\right)$. Cultured DRG neurons from WT mice rapidly responded to 32 -mer peptide, as indicated by increased $\left[\mathrm{Ca}^{2+}\right]_{\mathrm{i}}$ in $23 \%$ of neurons, suggesting that a subpopulation of DRG neurons express excitatory receptors for this protein fragment (Table 1 and Supplemental Figure 1A; supplemental material available online with this article; https://doi.org/10.1172/jci. insight.95704DS1). Scrambled control peptide induced responses in $6 \%$ of neurons (Table 1). All 32-mer responses were seen in small- to medium-diameter neurons, consistent with the size of nociceptors (Supplemental Figure 1B). In addition, the majority of neurons that responded to the 32-mer peptide also responded to capsaicin (55 of 71 neurons, $77 \%$ ), demonstrating that a subset of nociceptors expressing TRPV1 (transient receptor potential cation channel subfamily V member 1) can respond to 32-mer. The synthetic TLR2 ligand Pam3CSK4 also induced responses in 12\% of neurons (9 of 76) (Supplemental Figure 1C), primarily in small- to medium-diameter, capsaicin-sensitive neurons. Finally, in DRG cultures from Tlr2-null mice, only 5\% of neurons responded to 32-mer, suggesting that excitation is dependent on this signaling pathway (Table 1). In order to show that 32-mer responses are not an artifact of cell culture, we also performed calcium imaging using intact DRG from Pirt-GCaMP3-expressing mice $(16,25)$. In 3 intact DRG explants, $6 \%$ of capsaicin-sensitive neurons responded to 32-mer peptide, while scrambled peptide and vehicle solution each elicited responses in only $2 \%$ of capsaicin-sensitive neurons $(P=0.037)$ (Table 2 and Supplemental Figure 1D), supporting the cell culture findings.

In order to examine the potential proalgesic effects of 32-mer signaling in DRG neurons, we stimulated cultured DRG cells from WT mice with 32-mer peptide and measured release of the chemokine monocyte chemoattractant protein-1 (MCP-1/CCL2) into culture medium. We previously found that CCL2 is upregulated by DRG neurons in experimental osteoarthritis induced by DMM surgery and acts as a key

Table 2. Intracellular calcium responses in DRG neurons within intact explants

\begin{tabular}{|c|c|c|}
\hline & No. neuronal responses/total no. capsaicin-sensitive neurons (\%) & $P$ value ${ }^{A}$ \\
\hline Pirt-GCaMP3: 32-mer peptide (10 $\mu \mathrm{M})$ & $12 / 204(6 \%)$ & $P=0.037$ \\
\hline Pirt-GCaMP3: scrambled peptide $(10 \mu \mathrm{M})$ & $4 / 204(2 \%)$ & \\
\hline Pirt-GCaMP3: vehicle & $4 / 204(2 \%)$ & \\
\hline
\end{tabular}


A
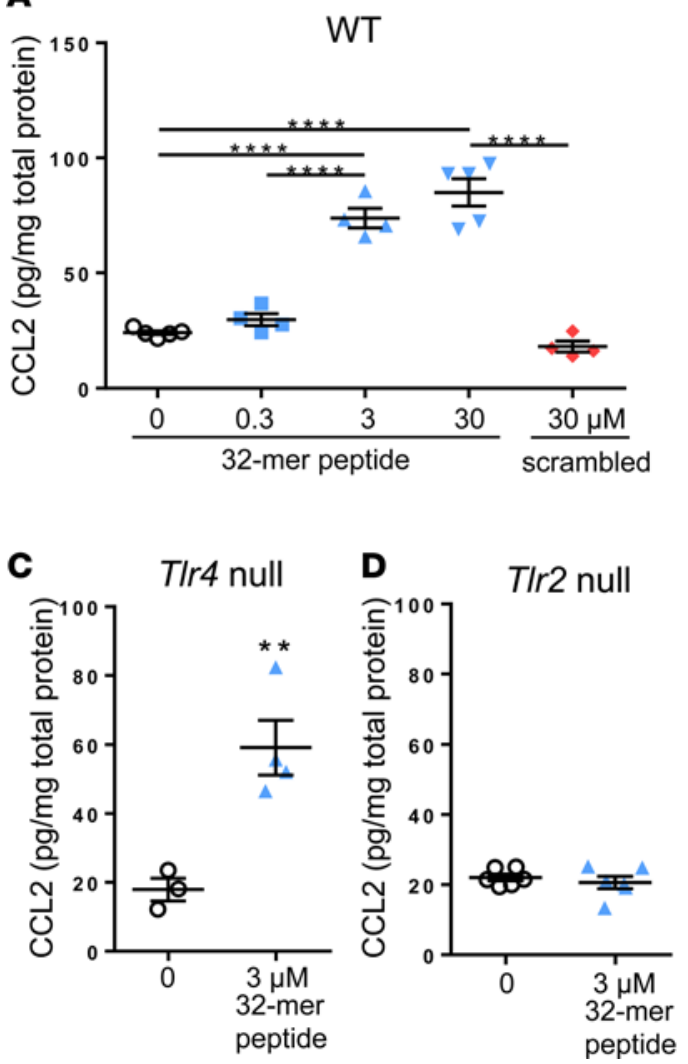
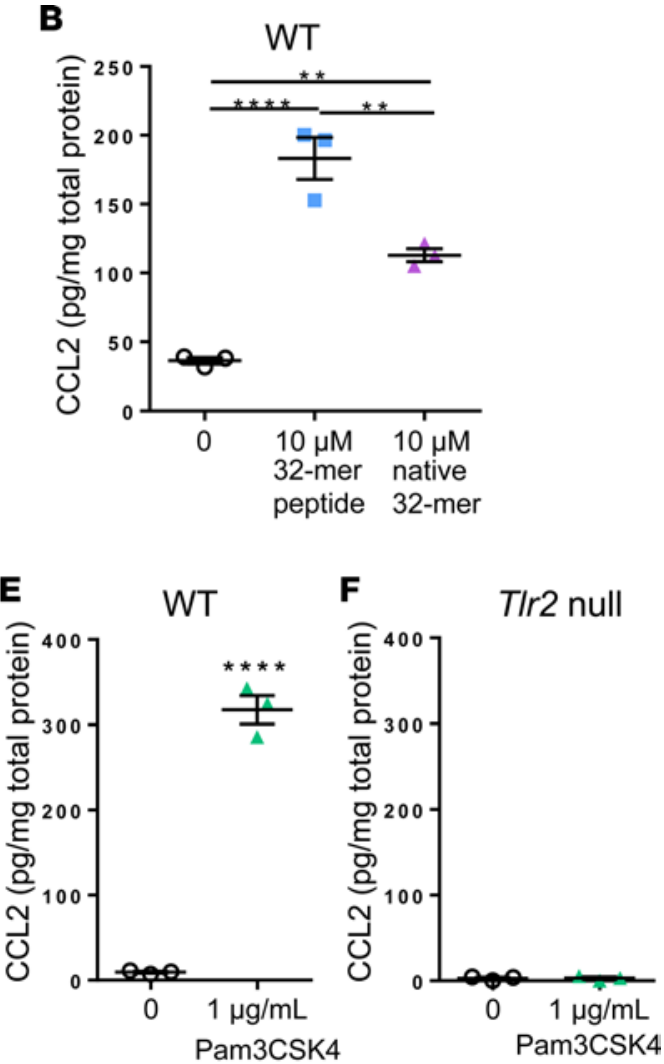

Figure 1. The 32-mer upregulates CCL2 protein production through TLR2 in cultured dorsal root ganglion (DRC) cells. (A) WT DRG cells were stimulated with 0-30 $\mu \mathrm{M}$ synthetic 32-mer or scrambled peptide. Repeated 0 vs. $3 \mu \mathrm{M}$ synthetic 32-mer in 4 independent cultures. (B) WT DRG cells were stimulated with $10 \mu \mathrm{M}$ synthetic or native porcine $32-$ mer in the presence of $30 \mu \mathrm{g} / \mathrm{ml}$ polymyxin-B. Representative of two independent experiments. (C) T/r4 ${ }^{-/-}$DRG

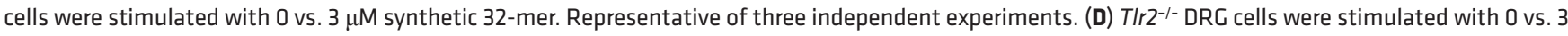
$\mu \mathrm{M}$ synthetic 32-mer. Representative of 3 independent experiments. (E) WT DRG cells were stimulated with $0 \mathrm{vs.} 1 \mu \mathrm{g} / \mathrm{ml}$ synthetic TLR2 ligand Pam3CSK4. Representative of 3 independent experiments. (F) T/r2 ${ }^{-1-}$ DRG cells were stimulated with 0 vs. $1 \mu \mathrm{g} / \mathrm{ml}$ synthetic TLR2 ligand Pam3CSK4. Representative of two independent experiments. For $\mathbf{A}$ and B: 1-way ANOVA with Bonferroni's post hoc tests; for C-F: unpaired 2-tailed Student's $t$ tests assuming equal variances; for $\mathbf{A}-\mathbf{F}:{ }^{* *} P<0.01,{ }^{* * *} P<0.0001$; mean \pm SEM

mediator of osteoarthritis pain (26). Overnight incubation of cultured DRG cells with 3 or $30 \mu \mathrm{M} 32$-mer peptide resulted in significant upregulation in CCL2 production compared with unstimulated cells (3.1-fold $[3 \mu \mathrm{M}]$ and 3.5-fold $[30 \mu \mathrm{M}], P<0.0001$ ) (Figure 1A). The highest concentration of scrambled peptide (30 $\mu \mathrm{M})$ did not induce CCL2 production (0.8-fold, $P>0.9999$ vs. unstimulated) (Figure 1A). A time-course experiment demonstrated that this upregulation in CCL2 release began after 6 hours of stimulation with 32-mer peptide (Supplemental Figure 2). Since this portion of the aggrecan molecule can be modified by keratan sulfate chains (27-30), we next tested whether the glycosylated native aggrecan 32-mer fragment can also stimulate DRG cells using native 32-mer purified from porcine cartilage. We found that native 32-mer stimulated DRG cells to produce elevated levels of CCL2 compared with unstimulated cells (3.1fold $[10 \mu \mathrm{M}], P<0.01$ ) (Figure $1 \mathrm{~B}$ ).

Which receptors mediate the effects of the 32-mer on DRG nociceptors? In order to answer this question, we prepared DRG cultures from Tlr2-null or Tlr4-null mice. Stimulation with 32-mer peptide $(3 \mu \mathrm{M})$ produced increased CCL2 in Tlr4-null (3.3-fold, $P<0.01$ ) (Figure 1C) but not in Tlr2-null DRG cultures (0.9-fold, $P=0.5$ ) (Figure 1D), suggesting that these effects are mediated through the activation of TLR2. WT DRG cells also responded to Pam3CSK4 (10 $\mu \mathrm{M}, 7.7$-fold, $P<0.0001)$ (Figure 1E), while Tlr2-null DRG cells did not (Figure 1F), providing additional evidence that TLR2 signaling can lead to increased CCL2 expression by these neurons. TLR2 is known to be expressed in a variety of cells as a functional heterodimer with either TLR1 or TLR6, but no reports have demonstrated expression by DRG neurons (15). Therefore, we immunostained DRG sections from WT naive mice and found that $17 \% \pm 2 \%$ of all DRG neurons stained positive for both TLR1 and TLR2, and $12 \% \pm 2 \%$ of all DRG neurons stained positive 

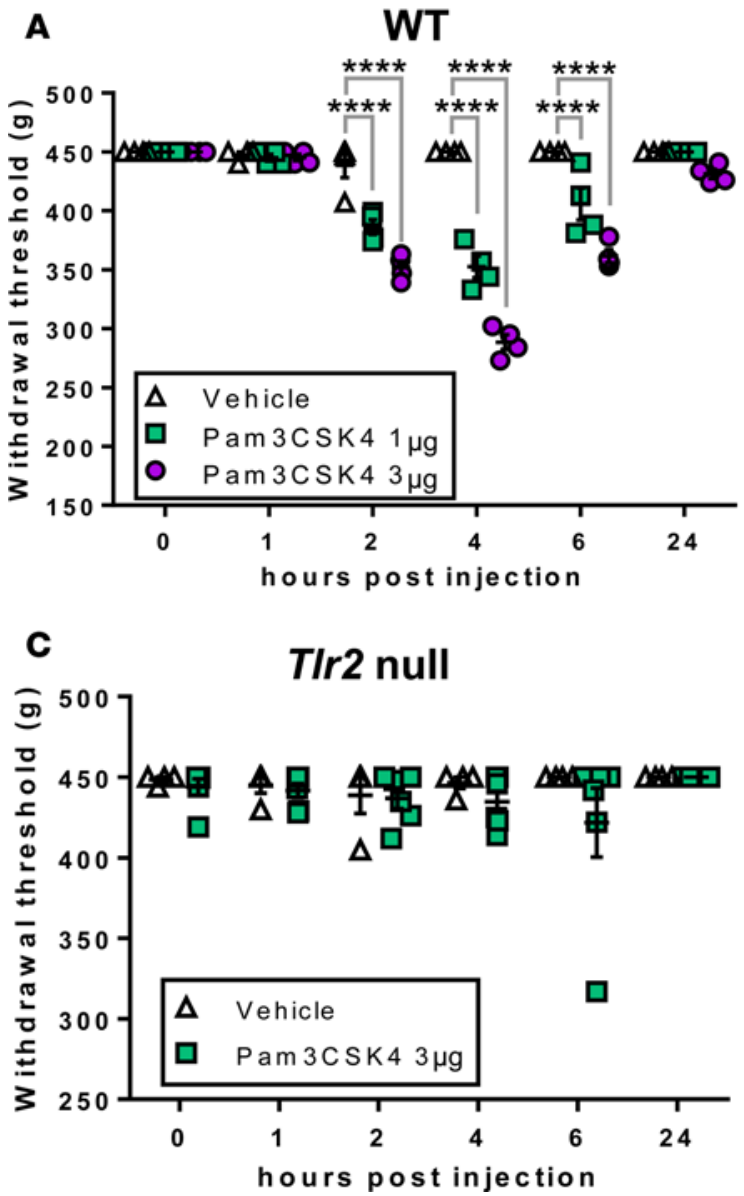

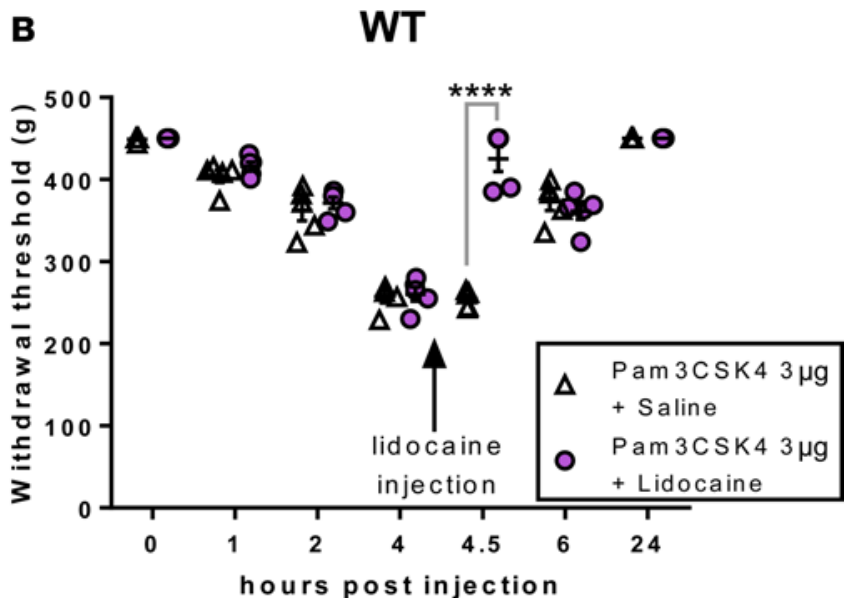

Figure 2. Intra-articular injection of synthetic TLR2 ligand, Pam3CSK4, induces knee hyperalgesia in naive mice. (A) Intra-articular injection of vehicle, 1 or $3 \mu \mathrm{g}$ Pam3CSK4 in WT mice. $n=4$ mice/treatment. (B) Intra-articular injection of $3 \mu \mathrm{g}$ Pam3CSK4 in WT mice followed by a second intra-articular injection of vehicle or lidocaine $(20 \mathrm{mg} / \mathrm{kg}) . n=5$ mice/treatment. (C) Intra-articular injection of vehicle or $3 \mu \mathrm{g}$ Pam3CSK4 in TIr2 ${ }^{-1-}$ mice. $n=4 /$ vehicle; $n=6 /$ Pam3CSK4. For A-C: Representative plot of 2 independent experiments; repeated-measures 2-way ANOVA with Bonferroni's post hoc tests was used to compare vehicle vs. drug at each time point; ${ }^{* * *} P<0.0001$; mean \pm SEM.

for both TLR6 and TLR2 (Supplemental Figure 3). In addition, a subset of TRPV1 neurons (marker for a subset of nociceptors) also stained positive for TLR2 (Supplemental Figure 3). Additional higher-magnification images are shown in Supplemental Figure 4, and negative control staining in Tlr2-null mice is shown in Supplemental Figure 5. In addition, naive Tlr2-lac $Z^{+/-}$reporter mice also demonstrated TLR2 expression in DRG neurons (Supplemental Figure 6).

Next, we aimed to determine whether the 32-mer has the potential to generate pain in vivo. We first injected Pam3CSK4 into the knee cavity of naive WT mice, which caused knee hyperalgesia in a dose-dependent fashion (Figure 2A and Supplemental Figure 7A). An intra-articular injection of lidocaine, administered once peak Pam3CSK4-induced knee hyperalgesia was established, reversed knee hyperalgesia, indicating a direct effect of peripheral nerves in mediating the observed hyperalgesia (Figure 2B and Supplemental Figure 7B). Tlr2-null mice did not develop knee hyperalgesia following injection of Pam3CSK4 into the knee cavity, indicating that hyperalgesia is indeed induced through the TLR2 pathway (Figure 2C and Supplemental Figure 7C). Next, we directly tested the effects of the 32-mer by injecting either the 32-mer or scrambled peptides into the knee cavity of naive mice. We observed that the 32-mer but not the scrambled peptide induced acute knee hyperalgesia in WT (Figure 3, A and B) but not Tlr2-null mice (Figure 3B and Supplemental Figure 8A). Additionally, we performed a dose-response experiment, which demonstrated that as little as $2.5 \mu \mathrm{g}$ of 32-mer peptide can stimulate knee hyperalgesia (Supplemental Figure 8B).

These data indicate that activation of TLR2 receptors by the 32-mer can elicit acute pain. In order to determine whether this process contributes to pain in active osteoarthritis, we explored the role of the 32-mer in generating knee hyperalgesia in an experimental model of osteoarthritis induced by DMM surgery. We have previously validated the DMM model as a suitable preclinical model that captures the long-term progression of osteoarthritis and associated pain $(26,31)$. We performed DMM surgery in WT, Chloe, and Tlr2null mice. Chloe mice are a transgenic line in which the MMP cleavage site $\left(\mathrm{N}^{341} \downarrow^{342} \mathrm{~F}\right)$ in the aggrecan interglobular domain is eliminated by amino acid changes, thus preventing production of the 32-mer fragment (32). However, since the aggrecanase cleavage site $\left(E^{373} \downarrow^{374} \mathrm{~A}\right)$ in the interglobular domain is not modified, 

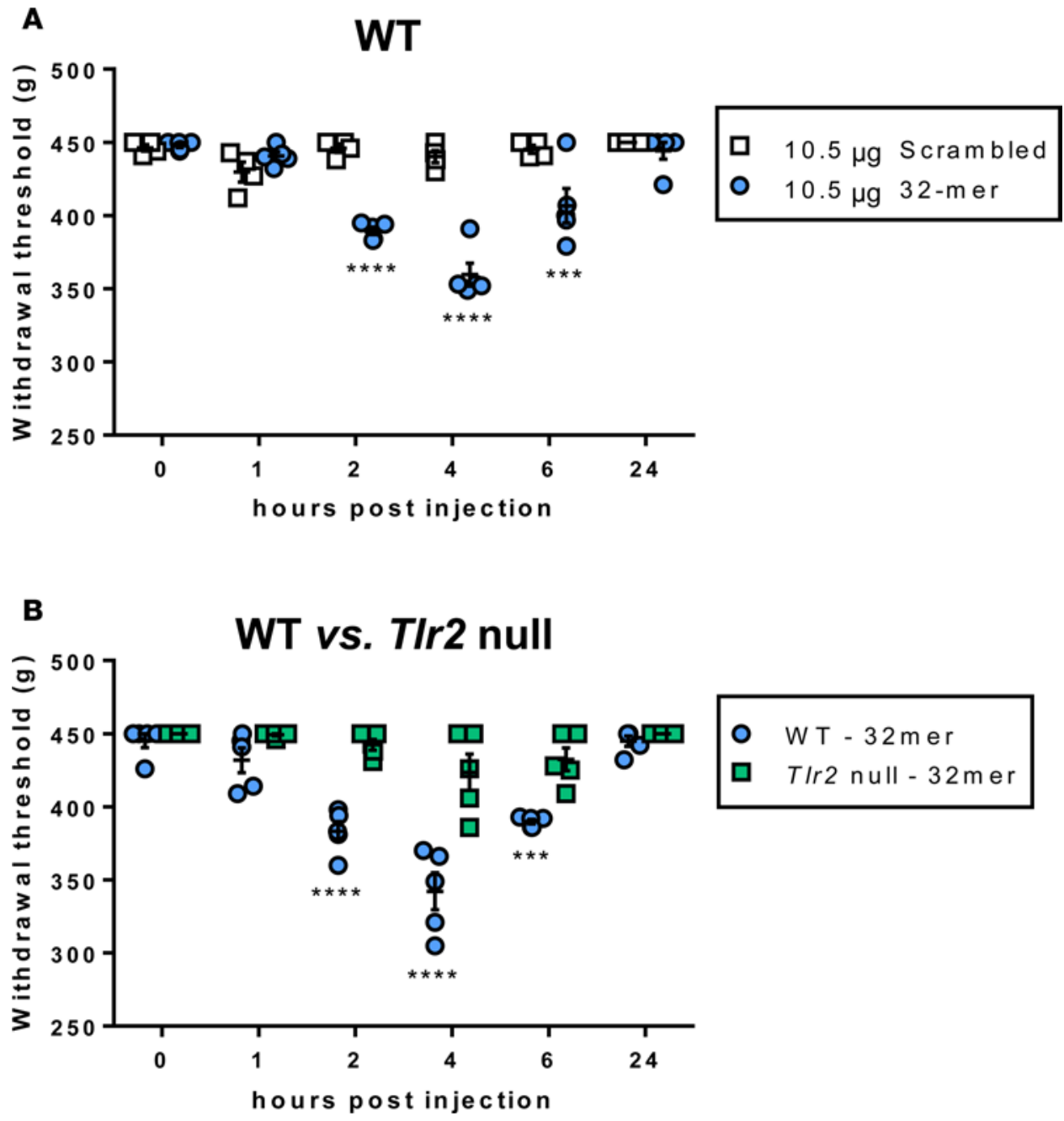

Figure 3. Intra-articular injection of synthetic 32-mer induces knee hyperalgesia in naive mice. (A) Intra-articular injection of $10.5 \mu \mathrm{g}$ synthetic 32 -mer or $10.5 \mu$ scrambled peptide in WT mice. $n=4 /$ scrambled; $n=5 / 32$-mer. Representative plot of 2 independent experiments. (B) Intra-articular injection of $10.5 \mu$ s synthetic 32-mer peptide in WT or TIr2-- mice; representative plot of 2 independent experiments. $n=5 /$ strain. For $\mathbf{A}$ and $\mathbf{B}$ : repeated-measures 2-way ANOVA with Bonferroni's post hoc tests was used to compare groups at each time point; ${ }^{* *} P<0.001,{ }^{* * *} P<0.0001 ;$ mean \pm SEM.

Chloe mice still develop osteoarthritis after DMM surgery (6). In this study, Chloe mice developed more cartilage degeneration and larger osteophytes than WT mice 4 and 16 weeks after surgery (Supplemental Figure 9, A and B). Subchondral bone sclerosis was also increased in Chloe mice compared with WT mice 4 weeks after DMM (Supplemental Figure 9C). Similar synovial changes were seen in both WT and Chloe mice throughout the 16 weeks (Supplemental Figure 9D). WT and Tlr2-null mice developed similar joint damage by 16 weeks after DMM (Supplemental Figure 9).

Similar to our previous results (33), DMM surgery caused pronounced knee hyperalgesia in WT mice by 2 weeks after surgery compared with sham-operated mice, and hyperalgesia slowly resolved through 16 weeks (Figure 4A). Four weeks after DMM, intra-articular injection of lidocaine reversed knee hyperalgesia (Figure 4B), indicating that it is a locally generated pain-related behavior. We have also previously demonstrated that knee hyperalgesia is reversed by systemic injection of morphine (33). In contrast, Chloe mice were protected from developing knee hyperalgesia until 12 weeks after DMM surgery compared with WT mice (Figure 4C and Supplemental Figure 10A), suggesting that the 32-mer fragment mediates the development of knee hyperalgesia. 32-mer peptide injected into the knee joint of naive Chloe mice induced knee hyperalgesia similar to that in WT naive mice (Figure 4D, Figure 3A, and Supplemental Figure 10B), confirming that these mice retain the ability to respond to the 32-mer. Tlr2-null mice were also protected from knee hyperalgesia up to 16 weeks after DMM surgery (Figure 4E), supporting the hypothesis that 32-mer signaling through TLR2 activation plays a key role in mediating early-phase knee hyperalgesia in this model. 

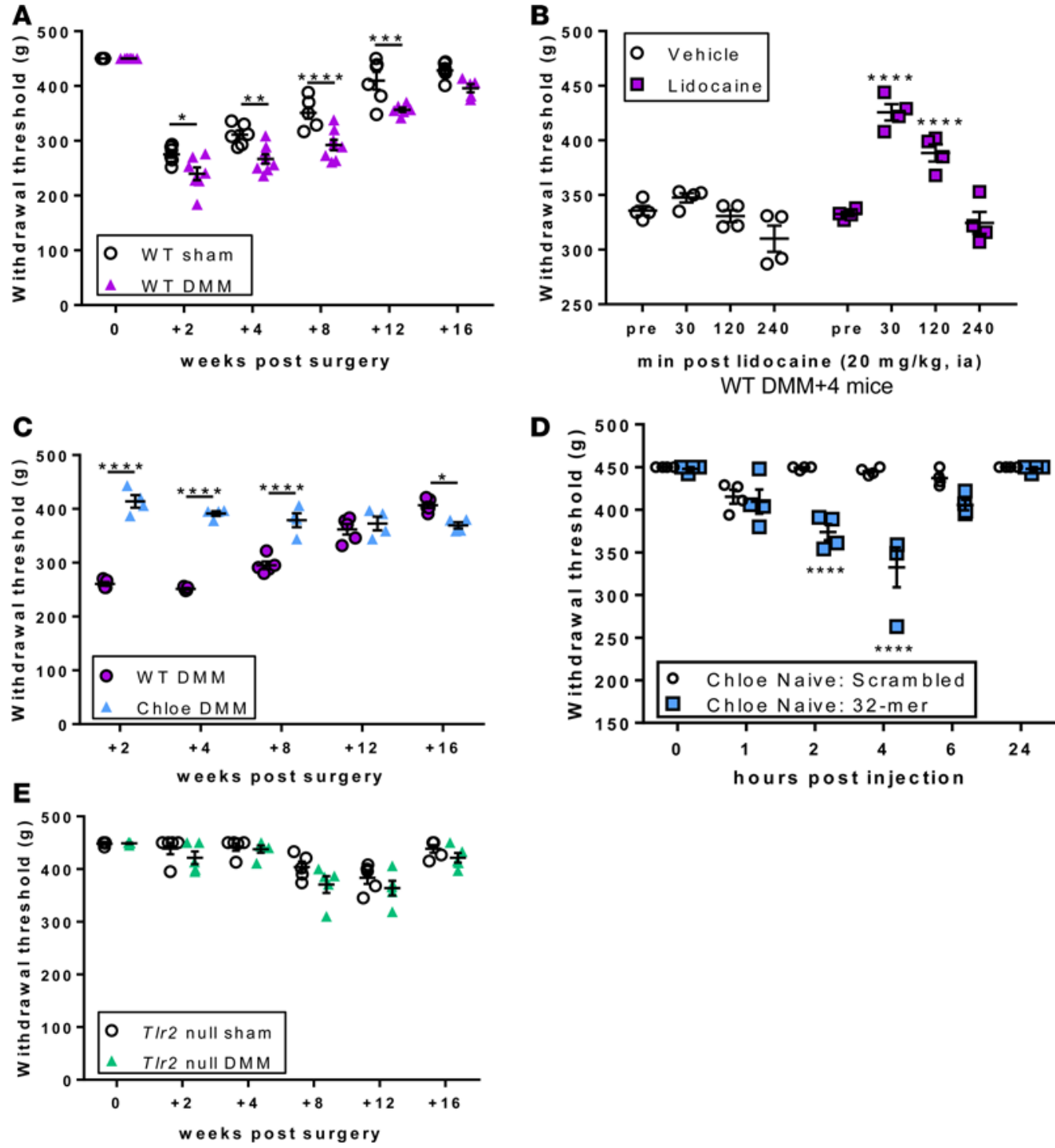

Figure 4. Blocking the production or action of the 32-mer protects against early knee hyperalgesia following destabilization of the medial meniscus (DMM) surgery. (A) Time course of knee hyperalgesia in WT mice following DMM surgery. $n=6-8$ mice/group. (B) Intra-articular (ia) injection of vehicle or lidocaine $(20 \mathrm{mg} / \mathrm{kg})$ four weeks after DMM surgery. $n=4$ mice/treatment. (C) Time course of knee hyperalgesia in WT $(n=5)$ vs. Chloe $(n=4$ mice) after DMM surgery. Repeated +2 to +8 week time points in 2 independent experiments. (D) Intra-articular injection of $10.5 \mu$ synthetic 32 -mer or scrambled peptide in naive Chloe mice. $n=4$ /group. Representative plot of 2 independent experiments. (E) Time course of knee hyperalgesia in $\mathrm{Tlr}^{-/-}$mice following sham or DMM surgery. $n=5$ mice/group. For A: Two-way ANOVA with Bonferroni's post hoc tests was used to compare responses at each time point; for B-E: Repeated measures 2-way ANOVA with Bonferroni's post hoc tests was used to compare groups at each time point. For $\mathbf{A}-\mathbf{E}$ : ${ }^{*} P<0.05$, ${ }^{* *} P<$ $0.01,{ }^{* *} P<0.001,{ }^{* * *} P<0.0001$; mean \pm SEM.

Finally, in order to test whether the 32-mer plays a role in mediating other DMM-associated chronic pain behaviors, we tested whether mechanical allodynia of the ipsilateral hindpaw was reduced after blocking the creation or action of the 32-mer. We found that WT, Chloe, and Tlr2-null mice developed similar patterns of mechanical allodynia in the ipsilateral hindpaw through 16 weeks after DMM (Supplemental Figure 11).

\section{Discussion}

In this study, we found that a specific fragment of aggrecan, the 32-mer, generated by the orchestrated action of ADAMTS- and MMP-mediated cleavage of the aggrecan core protein, directly excites DRG sensory neurons through TLR2. We have demonstrated for the first time to our knowledge that TLR2 can be coexpressed primarily with TLR1 and to a lesser extent with TLR6 by DRG sensory neurons; it thus 
joins the list of other TLRs (TLR3, TLR4, TLR5, TLR7, and TLR9) that have been shown to be expressed by DRG sensory neurons (15-17). Through the TLR2 pathway, the 32-mer aggrecan fragment induced knee hyperalgesia when injected intra-articularly into the knees of naive mice. Additionally, blocking the production of the 32-mer aggrecan fragment by using transgenic Chloe mice, or blocking the action of the 32-mer aggrecan fragment by using Tlr2-null mice, prevented knee hyperalgesia associated with the DMM model of osteoarthritis. A select group of extracellular matrix molecules, including low-molecular-weight hyaluronan, fibronectin-EDA, fragments of fibronectin, tenascin C, and biglycan, have been shown to act as DAMPs, signaling through TLR2 and TLR4 on non-neuronal cells (34). Recently, we added the 32-mer fragment of aggrecan to this list, by demonstrating that it can upregulate catabolic signaling through TLR2 expressed by chondrocytes, synovial fibroblasts, and macrophages (13). Chondroitin sulfate proteoglycans, including aggrecan, have been shown to play a role in guiding the development and growth of the sensory nervous system as well as regeneration following injury (35). Here, we show that an extracellular matrix fragment, specifically, the 32-mer aggrecan fragment, can signal through TLR2 on sensory neurons to cause hyperalgesia. A limitation of this work is that we did not conditionally delete TLR2 on sensory neurons, so it is possible that additional cell types also contribute to the observed pain phenotype. It will be interesting to investigate the effects of other extracellular matrix fragments on sensory neurons in future work.

Interestingly, we found that blocking the production of the 32-mer aggrecan fragment by using the transgenic Chloe mice was able to prevent knee hyperalgesia following DMM surgery, but not mechanical allodynia of the hind paw. This result suggests that in the DMM model of osteoarthritis, the primary action of the 32-mer aggrecan fragment on pain is locally within the knee joint, where it is likely being produced as part of the cartilage breakdown process associated with osteoarthritis.

Additionally, we demonstrated that Chloe mice are not protected from joint damage 16 weeks following DMM surgery, confirming the original report that showed lack of protection 8 weeks after DMM (6) (Supplemental Figure 4). This is consistent with the fact that cleavage at the aggrecanase-cleavage site $\left(\mathrm{E}^{373} \downarrow^{374} \mathrm{~A}\right)$ in the interglobular domain, and not the MMP cleavage site $\left(\mathrm{N}^{341} \downarrow^{342} \mathrm{~F}\right)$, has been shown to be key for promoting cartilage damage (6). Together, these results suggest that blocking the 32-mer aggrecan fragment alone is unlikely to be beneficial for stopping structural progression of osteoarthritis. On the other hand, blocking the 32-mer aggrecan fragment by using Chloe mice is sufficient to block knee hyperalgesia, which is a pain behavior associated with the early to mid-phase of the DMM model (33). Therefore, the 32-mer aggrecan fragment may represent a novel analgesic target in a subset of osteoarthritis patients, such as the post-traumatic joint injury population that can be identified early in the disease process.

In conclusion, we have demonstrated that activation of TLR 2 receptors expressed by nociceptors can promote pro-algesic signaling, particularly the production of the chemokine CCL 2 by these neurons, which we have previously identified as an important mediator of osteoarthritis pain (26). Our findings suggest that the 32-mer fragment of aggrecan directly activates TLR2 expressed by nociceptors within the knee joint, driving the development of osteoarthritis-associated knee hyperalgesia. These results provide new evidence indicating that molecules derived from the process of ongoing cartilage degradation can directly act upon nociceptors, thereby integrating osteoarthritis pain with other aspects of joint degeneration.

\section{Methods}

Additional methods are provided in Supplemental Methods.

Statistics. For calcium imaging experiments, $\chi^{2}$ tests were used to compare the number of responses. For CCL2 stimulation experiments, 1-way ANOVA with Bonferroni's post hoc tests or unpaired 2-tailed Student's $t$ tests assuming equal variances were used to compare the groups of interest. For knee hyperalgesia experiments tracking the same animals over time, a repeated-measures 2-way ANOVA with Bonferroni's post hoc tests was used to compare groups at each time point. For other knee hyperalgesia time courses, a 2-way ANOVA with Bonferroni's post hoc tests was used to compare responses at each time point. For mechanical allodynia time courses, 1-way ANOVA with Bonferroni's post hoc tests was used to compare each time point to time 0. For knee histopathology, data were analyzed using 2-way ANOVA with Bonferroni's post hoc tests or Mann-Whitney $U$ test. All analyses were carried out using GraphPad Prism version 6.07 for Windows (GraphPad Software). Results are presented as mean \pm SEM or median \pm interquartile range (IQR), as indicated. A $P$ value less than 0.05 was considered significant for all tests.

Study approval. All experiments were approved by the Rush University Institutional Animal Care and Use Committee. 


\section{Author contributions}

REM, AMM, and RJM were responsible for the overall study design. REM performed calcium imaging and CCL2 experiments, collected knee joints for histopathology, and performed statistical analyses. SI performed DMM surgery and assessed knee hyperalgesia and mechanical allodynia, and other in vivo techniques. PBT performed the immunohistochemistry. SBG, KL and AJF supplied the synthetic 32-mer peptide, native 32-mer fragment, and Chloe mice. AJF also provided input on overall experimental design and analysis of the data. The manuscript was written by REM, RJM, and AMM. All authors were involved in the design of the parts of the study they executed, discussed the design and results, commented on the manuscript and approved the final version.

\section{Acknowledgments}

REM was supported by the NIH/National Institute of Arthritis and Musculoskeletal and Skin Diseases (NIAMS) (F32AR062927 and K01AR070328). AMM (R01AR064251 and R01AR060364) and RJM (R01AR064251) were supported by NIAMS. AJF was supported by the National Health \& Medical Research Council (NHMRC) of Australia (no. 1060222).

Address correspondence to: Anne-Marie Malfait, 1611 W Harrison Street, Suite 510, Chicago, Illinois 60612, USA. Phone: 312.563.2925; Email: anne-marie_malfait@rush.edu. Or to: Rachel E. Miller, 1735 W Harrison Street, Room 714, Chicago, Illinois 60612, USA. Phone: 312.563.2021; Email: Rachel_Miller@rush.edu.

1. Ilic MZ, Handley CJ, Robinson HC, Mok MT. Mechanism of catabolism of aggrecan by articular cartilage. Arch Biochem Biophys. 1992;294(1):115-122.

2. Loulakis P, Shrikhande A, Davis G, Maniglia CA. N-terminal sequence of proteoglycan fragments isolated from medium of interleukin-1-treated articular-cartilage cultures. Putative site(s) of enzymic cleavage. Biochem J. 1992;284 (Pt 2):589-593.

3. Sandy JD, Neame PJ, Boynton RE, Flannery CR. Catabolism of aggrecan in cartilage explants. Identification of a major cleavage site within the interglobular domain. J Biol Chem. 1991;266(14):8683-8685.

4. Tortorella MD, et al. Purification and cloning of aggrecanase-1: a member of the ADAMTS family of proteins. Science. 1999;284(5420):1664-1666.

5. Abbaszade I, et al. Cloning and characterization of ADAMTS11, an aggrecanase from the ADAMTS family. J Biol Chem. 1999;274(33):23443-23450.

6. Little $\mathrm{CB}$, et al. Blocking aggrecanase cleavage in the aggrecan interglobular domain abrogates cartilage erosion and promotes cartilage repair. J Clin Invest. 2007;117(6):1627-1636.

7. Malfait AM, Liu RQ, Ijiri K, Komiya S, Tortorella MD. Inhibition of ADAM-TS4 and ADAM-TS5 prevents aggrecan degradation in osteoarthritic cartilage. J Biol Chem. 2002;277(25):22201-22208.

8. Apte SS. Anti-ADAMTS5 monoclonal antibodies: implications for aggrecanase inhibition in osteoarthritis. Biochem J. 2016;473(1):e1-e4.

9. Gilbert AM, Bikker JA, O'Neil SV. Advances in the development of novel aggrecanase inhibitors. Expert Opin Ther Pat. 2011;21(1):1-12.

10. Miller RE, Tran PB, Ishihara S, Larkin J, Malfait AM. Therapeutic effects of an anti-ADAMTS-5 antibody on joint damage and mechanical allodynia in a murine model of osteoarthritis. Osteoarthr Cartil. 2016;24(2):299-306.

11. Fosang AJ, Neame PJ, Hardingham TE, Murphy G, Hamilton JA. Cleavage of cartilage proteoglycan between G1 and G2 domains by stromelysins. J Biol Chem. 1991;266(24):15579-15582.

12. Fosang AJ, Last K, Gardiner P, Jackson DC, Brown L. Development of a cleavage-site-specific monoclonal antibody for detecting metalloproteinase-derived aggrecan fragments: detection of fragments in human synovial fluids. Biochem J. 1995;310 (Pt 1):337-343.

13. Lees S, et al. Bioactivity in an aggrecan 32-mer fragment is mediated via Toll-like receptor 2. Arthritis Rheumatol. 2015;67(5):1240-1249.

14. Hayward JH, Lee SJ. A decade of research on TLR2 discovering its pivotal role in glial activation and neuroinflammation in neurodegenerative diseases. Exp Neurobiol. 2014;23(2):138-147.

15. Liu T, Gao YJ, Ji RR. Emerging role of Toll-like receptors in the control of pain and itch. Neurosci Bull. 2012;28(2):131-144.

16. Miller RE, et al. Damage-associated molecular patterns generated in osteoarthritis directly excite murine nociceptive neurons through Toll-like receptor 4. Arthritis Rheumatol. 2015;67(11):2933-2943.

17. Xu ZZ, et al. Inhibition of mechanical allodynia in neuropathic pain by TLR5-mediated A-fiber blockade. Nat Med. 2015;21(11):1326-1331.

18. Kim C, et al. Antagonizing neuronal Toll-like receptor 2 prevents synucleinopathy by activating autophagy. Cell Rep 2015;13(4):771-782.

19. Kim D, et al. A critical role of toll-like receptor 2 in nerve injury-induced spinal cord glial cell activation and pain hypersensitivity. J Biol Chem. 2007;282(20):14975-14983.

20. Kim D, You B, Lim H, Lee SJ. Toll-like receptor 2 contributes to chemokine gene expression and macrophage infiltration in the dorsal root ganglia after peripheral nerve injury. Mol Pain. 2011;7:74.

21. Diogenes A, Ferraz CC, Akopian AN, Henry MA, Hargreaves KM. LPS sensitizes TRPV1 via activation of TLR4 in trigeminal 
sensory neurons. J Dent Res. 2011;90(6):759-764.

22. Qi J, et al. Painful pathways induced by TLR stimulation of dorsal root ganglion neurons. J Immunol. 2011;186(11):6417-6426.

23. Allette YM, et al. Identification of a functional interaction of HMGB1 with receptor for advanced glycation end-products in a model of neuropathic pain. Brain Behav Immun. 2014;42:169-177.

24. Shibasaki M, et al. Induction of high mobility group box-1 in dorsal root ganglion contributes to pain hypersensitivity after peripheral nerve injury. Pain. 2010;149(3):514-521.

25. Kim YS, et al. Central terminal sensitization of TRPV1 by descending serotonergic facilitation modulates chronic pain. Neuron. 2014;81(4):873-887.

26. Miller RE, et al. CCR2 chemokine receptor signaling mediates pain in experimental osteoarthritis. Proc Natl Acad Sci USA 2012;109(50):20602-20607.

27. Barry FP, Gaw JU, Young CN, Neame PJ. Hyaluronan-binding region of aggrecan from pig laryngeal cartilage. Amino acid sequence, analysis of N-linked oligosaccharides and location of the keratan sulphate. Biochem J. 1992;286 (Pt 3):761-769.

28. Brown GM, Huckerby TN, Bayliss MT, Nieduszynski IA. Human aggrecan keratan sulfate undergoes structural changes during adolescent development. J Biol Chem. 1998;273(41):26408-26414.

29. Flannery CR, Little CB, Caterson B. Molecular cloning and sequence analysis of the aggrecan interglobular domain from porcine, equine, bovine and ovine cartilage: comparison of proteinase-susceptible regions and sites of keratan sulfate substitution. Matrix Biol. 1998;16(8):507-511.

30. Fosang AJ, Last K, Poon CJ, Plaas AH. Keratan sulphate in the interglobular domain has a microstructure that is distinct from keratan sulphate elsewhere on pig aggrecan. Matrix Biol. 2009;28(1):53-61.

31. Malfait AM, et al. ADAMTS-5 deficient mice do not develop mechanical allodynia associated with osteoarthritis following medial meniscal destabilization. Osteoarthr Cartil. 2010;18(4):572-580.

32. Little $\mathrm{CB}$, et al. Matrix metalloproteinases are not essential for aggrecan turnover during normal skeletal growth and development. Mol Cell Biol. 2005;25(8):3388-3399.

33. Miller RE, et al. Chemogenetic Inhibition of pain neurons in a mouse model of osteoarthritis. Arthritis Rheumatol. 2017;69(7):1429-1439.

34. Sokolove J, Lepus CM. Role of inflammation in the pathogenesis of osteoarthritis: latest findings and interpretations. Ther $A d v$ Musculoskelet Dis. 2013;5(2):77-94.

35. Gardiner NJ. Integrins and the extracellular matrix: key mediators of development and regeneration of the sensory nervous system. Dev Neurobiol. 2011;71(11):1054-1072. 